\title{
Remarks on the Chern-Simons photon term in the QED description of graphene
}

\author{
David Dudal, ${ }^{1,2, *}$ Ana Júlia Mizher, ${ }^{1,3, \dagger}$ and Pablo Pais ${ }^{1,4, \$}$ \\ ${ }^{1}$ KU Leuven Campus Kortrijk-Kulak, Department of Physics, \\ Etienne Sabbelaan 53 bus 7657, 8500 Kortrijk, Belgium \\ ${ }^{2}$ Ghent University, Department of Physics and Astronomy, Krijgslaan 281-S9, 9000 Gent, Belgium \\ ${ }^{3}$ Instituto de Física Teórica, Universidade Estadual Paulista, Rua Dr. Bento Teobaldo Ferraz, \\ 271-Bloco II, 01140-070 Sáo Paulo, SP, Brazil \\ ${ }^{4}$ Faculty of Mathematics and Physics, Charles University, \\ V Holešovičkách 2, 18000 Prague 8, Czech Republic
}

(Received 26 June 2018; published 10 September 2018)

\begin{abstract}
We revisit the Coleman-Hill theorem in the context of reduced planar QED. Using the global U(1) Ward identity for this nonlocal but still gauge-invariant theory, we can confirm that the topological piece of the photon self-energy at zero momentum does not receive further quantum corrections apart from the potential one-loop contribution, even when considering the Lorentz-noninvariant case due to the Fermi velocity $v_{F}<c$. This is of relevance to probe possible time parity odd dynamics in a planar sheet of graphene which has an effective description in terms of $(2+1)$-dimensional planar reduced QED.
\end{abstract}

DOI: 10.1103/PhysRevD.98.065008

\section{CONTEXT AND MOTIVATION}

Quantum electrodynamics in $(2+1)$ dimensions $\left(\mathrm{QED}_{3}\right)$ has been widely used as a toy model for quantum chromodynamics (QCD). This is due to the fact that although it is Abelian, $\mathrm{QED}_{3}$ exhibits similar features as non-Abelian gauge theories, making it possible, for instance, to map and investigate chiral symmetry breaking and confinement into it [1-5]. The similarity is reinforced by the fact that a non-Abelian gauge theory at high temperature suffers a dimensional reduction and, if coupled to $N_{f}$ fermion families, the non-Abelian interactions are suppressed by a factor of $N_{f}^{-1}$, so that in the large $N_{f}$ limit the theory can be considered approximately Abelian.

Recently, the emergence of the so-called Dirac and Weyl planar materials [6] converted $\mathrm{QED}_{3}$ into a playground in which a potential link between high energy physics (including quantum fields in curved spacetimes) and condensed matter can emerge [7-13]. Those are materials in which, due to the specific structure of their underlying lattice, the charge carriers present a relativistic-like behavior, correctly described by a Dirac-like equation in some

\footnotetext{
*david.dudal@kuleuven.be †ana.mizher@kuleuven.be pablo.pais@kuleuven.be
}

Published by the American Physical Society under the terms of the Creative Commons Attribution 4.0 International license. Further distribution of this work must maintain attribution to the author(s) and the published article's title, journal citation, and DOI. Funded by SCOAP. regimes. Particularly, the physical realization of graphene and other materials in two space dimensions, which are proven to contain a priori massless Dirac spinors, naturally yields the fermionic part of $\mathrm{QED}_{3}[14,15]$ through the continuum limit of the tight-binding theory, usually applied to describe their conduction electrons, which in turn implies a direct connection to QCD, as discussed above.

Nevertheless, even though in these systems the fermions are constrained to remain in-plane and therefore are correctly described by a theory in $(2+1)$ dimensions, the gauge fields responsible for the interaction between these electrons are not subject to the same constraint. One of the most remarkable consequences of this fact is that the interaction between electrons remains the familiar $\sim 1 / r$ potential rather than the logarithmic one that would take place if the gauge fields were also restricted to the plane. Therefore, it is convenient and necessary to modify $\mathrm{QED}_{3}$ in order to merge the desired features of the two sectors of the theory, starting with a general $(3+1)$ theory and dimensionally reducing it to a nonlocal effective $(2+1)$ theory. This procedure was followed in similar approaches in [16] with the so-called pseudo-QED (PQED), and posteriorly in [17], receiving the name of reduced QED (RQED). In this work we follow the outline of RQED, but both constructions are equivalent; for interesting applications of PQED, we refer to [18-21].

In the context of pure $\mathrm{QED}_{3}$, the most general structure of the action allows for a term in the gauge sector that breaks time reversal ( $\mathrm{T})$, namely, the Chern-Simons (CS) term. Its presence gives a mass to the photon $[22,23]$ and, for this reason, it is also known as a topological mass term 
(actually, in the Abelian case there is no real topology involved and the term "topological" is used for historical reasons based on its non-Abelian counterpart). This term is important in several contexts in condensed matter; for instance, it naturally leads to the transverse conductivity observed from the Hall effect, and it is crucial to model high $T_{c}$ superconductivity [24]. It was shown that radiative corrections coming from interaction terms can give a contribution for the topological photon mass up to one loop. Remarkably, a theorem by Coleman and Hill [25] demonstrates that, apart from one loop, all corrections to the topological mass term vanish identically to all orders. This was done on general grounds, considering the photon interacting with any massive scalar, spinor, or vector field with arbitrary gauge-invariant interactions. The massive nature of the field excitations interacting with the photon is crucial here, to avoid the typical infrared subtleties in lower-dimensional field theories. In particular, the Coleman-Hill theorem does not hold in the presence of massless degrees of freedom, as explicitly illustrated in e.g., [26]. Indeed, infrared singularities, typical for lowerdimensional field theories, can disturb the argument.

Regarding the importance of RQED in the description of planar Dirac systems in condensed matter, precisely for those systems that allow for a direct analogy with QCD, it is important to verify if the Coleman-Hill theorem also holds for this theory, in particular, when the Lorentz-noninvariant version of RQED is considered. In this work we demonstrate that higher order radiative corrections are exactly vanishing in $\mathrm{RQED}$, in the same way as for $\mathrm{QED}_{3}$, meaning that the topological photon parameter arises at one loop or does not arise at all. In Sec. II we discuss briefly how the tight-binding model yields $\mathrm{QED}_{3}$ in the continuum limit and present the general features of RQED, including its gauge invariance and freedom of gauge choice, before and after the reduction. In particular, we discuss possible mass terms for the fermions that are important if we want to apply our theory directly to graphene. The role of electromagnetic background fields in the radiative corrections, which is important in manipulations to study transport phenomena in materials, is also briefly highlighted, with explicit computations relegated to a future, longer paper. In Sec. III we prove in full detail, for the Lorentz invariant case, that corrections of order higher than one are null, thus motivating our choice of mass terms, from both the (crucially different) two- and four-component spinor viewpoints; finally, we summarize the explicit one-loop computation in the absence of background fields. Section IV is devoted to the generalization of the argument to the Lorentz noninvariant case. In Sec. V we present our final remarks.

\section{SETTING THE STAGE: PLANAR SYSTEMS AND RQED}

In this section we briefly review how the continuum limit of the tight-binding model describing graphene can be associated with QED in a lower dimension and why, in this case, it is interesting to work with a modified version of this theory, known as reduced QED. We discuss in some more detail the gauge invariance of this theory before and after the reduction, especially concerning the gauge fixing term, which is not covered so well in other papers. We pay particular attention to the role of fermion masses and how, in the continuum limit, different structures can result in equivalent mass terms, an issue that usually, although known [27], is undervalued in the literature. Finally, we deduce the photon propagator for RQED, taking into account a Chern-Simons term, and we discuss the role of its coefficient, the $\theta$ parameter. We compare it to the standard $\mathrm{QED}_{3}$, where $\theta$ is responsible for generating a photon mass, and we show that in RQED, although it also appears explicitly in the propagator, it differs dimensionally from a mass parameter; i.e., the photon remains massless for RQED.

As a starting point, we briefly present the very basics of graphene from a point of view that is convenient for a quantum field theoretical approach. Many excellent reviews are available on this subject-for instance $[14,15]$ and references therein. Graphene, constituted by a single sheet of carbon atoms tightly packed into a twodimensional honeycomb lattice, can be regarded in terms of two periodic sublattices $L_{A}$ and $L_{B}$. Here, we follow the convention of [14] (for an alternative convention see for instance [28]) and define the primitive two-dimensional vectors $\vec{a}_{i}$ for sublattice $L_{A}$ and $\vec{b}_{i}$ for the reciprocal sublattice, as $\vec{a}_{1}=a(1 / 2, \sqrt{3} / 2), \quad \vec{a}_{2}=a(1 / 2,-\sqrt{3} / 2)$, and $\vec{b}_{1}=\frac{2 \pi}{a}(1 / 2, \sqrt{3} / 2), \vec{b}_{2}=\frac{2 \pi}{a}(1 / 2,-\sqrt{3} / 2)$, where $a$ is the sublattice spacing. It is also convenient to introduce the three nearest-neighbor vectors $\vec{s}_{i}$,

$$
\begin{aligned}
& \vec{s}_{1}=a(0,1 / \sqrt{3}), \quad \vec{s}_{2}=a(1 / 2,-\sqrt{3} / 6), \\
& \vec{s}_{3}=a(-1 / 2,-\sqrt{3} / 6),
\end{aligned}
$$

where $\ell=\frac{a}{\sqrt{3}}$ is the minimal lattice length.

The inner orbitals are strongly bonded to their respective carbon atom, while the $\pi$ orbitals present a weak overlap. The electrons presented in these orbitals are called $\pi$ electrons. Following the usual tight-binding approach, only the interaction of each charge carrier with the nearest neighbors of $\pi$ electrons is considered. The Hamiltonian is written as

$\mathcal{H}=-t \sum_{\vec{r} \in L_{A}} \sum_{i=1}^{3}\left(a^{\dagger}(\vec{r}) b\left(\vec{r}+\vec{s}_{i}\right)+b^{\dagger}\left(\vec{r}+\overrightarrow{s_{i}}\right) a(\vec{r})\right)$,

where the first sum is only along sublattice $L_{A}, t$ is the nearest-neighbor hopping energy, and $a, a^{\dagger}\left(b, b^{\dagger}\right)$ are the anticommuting ladder operators in the sublattice $L_{A}\left(L_{B}\right)$. Applying a Fourier transformation, it is straightforward 
to compute the energy-momentum dispersion relation $[14,15]$ :

$$
\begin{aligned}
& E\left(k_{x}, k_{y}\right) \\
& \quad= \pm t \sqrt{3+2 \cos \left(\sqrt{3} k_{y} a\right)+4 \cos \left(\frac{\sqrt{3}}{2} k_{y} a\right) \cos \left(\frac{3}{2} k_{x} a\right)} .
\end{aligned}
$$

The valence and conduction bands, generated by the opposite signs in the dispersion relation, touch at six points (Dirac points), of which only two are inequivalent. Here we choose them to be $\vec{K}_{ \pm}= \pm 2 \pi / a(2 / 3,0)$. Expanding the expression above around these zero energy points, one can verify that the dispersion relation for each one of them is linear, $E_{ \pm}(\vec{p})= \pm \hbar v_{F}|\vec{p}|$. Here, the Fermi velocity is determined by $v_{F}=\frac{3}{2} t \ell=\frac{\sqrt{3}}{2} a t \approx \frac{c}{300}$. It was shown [29] that the annihilation operators $a$ and $b$ can be accommodated in a spinor field when we expand around the above Dirac points and, therefore, it can be seen as a relativisticlike fermion that obeys a Dirac-like equation. In conclusion, the continuum limit of the nearest-neighbor approach in a tight-binding model applied to a pure hexagonal sublattice with two intertwined triangular sublattices yields a massless version of the fermion sector of $\mathrm{QED}_{3}$.

Following this approach and working with $\eta_{\mu \nu}=$ $\operatorname{diag}(-1,1,1)$, the action of the system reads

$$
\mathcal{S}_{f}=\int \mathrm{d}^{3} x\left[\bar{\psi}\left[\gamma^{0}\left(i \partial_{0}-i v_{F} \vec{\gamma} \cdot \vec{\nabla}\right] \psi\right],\right.
$$

where only the first two spatial gamma matrices $\vec{\gamma}$ enter. Here we show explicitly the Fermi velocity $v_{F} \leq 1$ expressed in units of $c=1$. Later we deal with extra fields, in addition to the fermion description of the $\pi$ electrons. If only these fermions were taken into account, we could take a simpler action in a Minkowskian space with a velocity $v_{F}$ instead of $c[12,13,30,31]$. In what follows we first focus on the $v_{F}=1$ limiting case, i.e., the standard Lorentz-invariant Dirac action. In Sec. IV we generalize the construction to the $v_{F}<1$ case.

Interactions with external sources or alterations on the underlying lattice, for instance using a substrate or doping, could produce a gap between the bands. This can be represented at the level of the action by a specific Dirac mass term, $m \bar{\psi} \psi$, or interaction terms involving the matter current. Let us refer to $[14,32,33]$ for such possibilities and classification of the mass terms. Interaction terms that are bilinear in the fermion field will change the basic symmetries of the action, depending on their particular gamma matrix structure. In this paper we work in the chiral basis, where the gamma matrices and the fifth gamma matrix are given by

$$
\begin{array}{ll}
\gamma^{0}=\left(\begin{array}{cc}
0 & I_{2} \\
I_{2} & 0
\end{array}\right), & \gamma^{i}=\left(\begin{array}{cc}
0 & \sigma^{i} \\
-\sigma^{i} & 0
\end{array}\right), \\
\gamma^{5}=\left(\begin{array}{cc}
-I_{2} & 0 \\
0 & I_{2}
\end{array}\right), & i=1,2,3
\end{array}
$$

where $I_{2}$ is the $2 \times 2$ identity and $\sigma^{i}$ are the standard Pauli matrices. Among the several possibilities of interactions, one can observe that certain terms are completely equivalent to the Dirac mass term as they correspond to a change in the variables in the path integral. Since there is no axial anomaly in $(2+1)$ dimensions, the result must describe the same physics. This is the case for the (anti-Hermitian) mass terms $m \bar{\psi} \gamma^{3} \psi$ and $i m \bar{\psi} \gamma^{5} \psi$, which can be reached from the standard Dirac mass term by performing the following unitary transformations in the fermion fields [27], respectively:

$$
\begin{array}{ll}
\psi \rightarrow e^{i \beta \gamma^{5}} \psi ; & \bar{\psi} \rightarrow \bar{\psi} e^{i \beta \gamma^{5}} . \\
\psi \rightarrow e^{\alpha \gamma^{3}} \psi ; & \bar{\psi} \rightarrow \bar{\psi} e^{\alpha \gamma^{3}},
\end{array}
$$

with appropriate choices of the "angles" $\alpha$ and $\beta$. In the case of massless fermions, Eqs. (6a) and (6b) both constitute symmetries of the theory and are part of a larger $\mathrm{U}(2)$ invariance (see [14]).

We remark that this is a feature of the continuum limit, and discretization can bring differences between those terms. For example, the tight-binding lattice models that would induce the three masses are different [32,33], but they share their continuum limit. Notice also that all these masses correspond to a T-even sector [14], where we refer to T-even or T-odd in the four-component spinor language. In the two-component description the symmetry behavior of the fermion mass terms can be different (see [34,35]).

Considering these variations of the Dirac mass in the continuum, it is particularly useful to use $m \bar{\psi} \gamma^{3} \psi$ when working with a four-component representation of the fermion field since, in this way, it is possible to decompose and rewrite the action in terms of two decoupled twocomponent spinors. This point will be discussed in more detail below in Sec. III B. The subtle differences between both formulations can also be seen in [34].

Besides the variants of the Dirac mass, one other specific mass term is particularly important, the Haldane mass $m_{o} \gamma^{3} \gamma^{5}$ [36]. This term is totally independent of the masses previously discussed, as it corresponds to a T-odd bilinear term. It is especially interesting because in pure $\mathrm{QED}_{3}$ it can be directly related to the CS term.

The gauge sector of pure $\mathrm{QED}_{3}$ is described by

$$
\mathcal{S}_{\mathrm{QED}_{3}}=\int \mathrm{d}^{3} x\left[-\frac{1}{4} F_{\mu \nu} F^{\mu \nu}+\frac{1}{2 \xi}(\partial \cdot A)^{2}-\frac{\theta}{2} \epsilon^{\mu \nu \rho} A_{\mu} \partial_{\nu} A_{\rho}\right],
$$


where the first term is the usual Maxwell term, the second is a linear gauge fixing term, and the last one is the CS term. On one hand, the one-loop radiative corrections from a fermion with Haldane mass generate a T-odd piece in the photon polarization tensor [37,38], which can be translated into the presence of the CS term in the gauge sector of the action. The Coleman-Hill theorem [25] guarantees that no higher order corrections are allowed, so the connection of the two terms is clearly pictured. On the other hand, the presence of a CS term dynamically generates a Haldane mass for the fermions [39] already at one loop as well.

As discussed before, in order to correctly describe electrons confined to a plane but whose interaction is the usual Coulomb interaction, it is necessary to consider the gauge fields living in the three-dimensional spatial bulk rather than in the two-dimensional spatial plane. To obtain a consistent theory combining the suitable conditions for fermions and gauge fields, Refs. [16,17] start with the gauge theory in four dimensions and integrate out the gauge field. For the sake of brevity, we consider standard $\mathrm{QED}_{4}$ (without a Chern-Simons term) written as

$$
\mathcal{S}_{\mathrm{QED}_{4}}=\int \mathrm{d}^{4} x\left[-\frac{1}{4} F_{\mu \nu} F^{\mu \nu}+\frac{1}{2 \xi}(\partial \cdot A)^{2}+j_{\mu} A^{\mu}\right] .
$$

The Dirac matter currents are

$$
j^{\mu}= \begin{cases}i \bar{\psi} \gamma^{\mu} \psi \delta\left(x_{3}\right) & \text { for } \mu=0,1,2, \\ 0 & \text { for } \mu=3,\end{cases}
$$

with the fermion fields only dependent on $\left(x_{0}, x_{1}, x_{2}\right)$. This formally expresses the fact that the fermion dynamics is restricted to the $\left(x_{1}, x_{2}\right)$ plane, i.e., the planar graphene sheet. The current is conserved, $\partial_{\mu} j^{\mu}=0$. The easiest way to proceed is to Wick rotate to Euclidean space and to Fourier transform (denoted by the -notation throughout the remainder of the text) in order to integrate out the fourdimensional gauge field, leading to

$$
\mathcal{S}_{\text {eff }}=\int \mathrm{d}^{4} p\left[\hat{j}^{\mu} \hat{D}_{\mu \nu}^{T}\left(\vec{p}, p_{3}\right) \hat{j}^{\nu}\right],
$$

where $\vec{p}=\left(p_{0}, p_{1}, p_{2}\right)$. Note that $\hat{D}_{\mu \nu}^{T}\left(\vec{p}, p_{3}\right)=\left(\delta_{\mu \nu}-\right.$ $\frac{p_{\mu} p_{\nu}}{\left(\vec{p}^{2}+p_{3}^{2}\right)} \times \frac{1}{\left(\vec{p}^{2}+p_{3}^{2}\right)}$ is the (gauge-independent) transverse projection of the free photon propagator, which appears due to the conserved fermion current. As the Fouriertransformed currents will not depend on $p_{3}$, we can integrate out the latter, leading to

$$
\mathcal{S}_{\text {eff }}=\int \mathrm{d}^{3} p\left[\hat{j}^{\mu} \hat{\mathcal{D}}_{\mu \nu}^{T}(\vec{p}) \hat{j}^{\nu}\right] .
$$

The indices $\mu, \nu$ are, from now on, restricted to $x_{0}, x_{1}, x_{2}$, and we can forget about the $\delta\left(x_{3}\right)$ in the definition of the current $j_{\mu}$. Furthermore, we set

$$
\hat{\mathcal{D}}_{\mu \nu}^{T}(\vec{p})=\left(\delta_{\mu \nu}-\frac{p_{\mu} p_{\nu}}{p^{2}}\right) \frac{1}{2 p}, \quad p=\sqrt{\vec{p}^{2}} .
$$

It is worth noting that, in passing from (9) to (10), an irrelevant longitudinal term appearing in $\hat{j}_{\mu} \ldots \hat{j}_{\nu}$ has been dropped from (10). It is then easily recognized that the effective action (10) can be equivalently reformulated in terms of a Euclidean nonlocal gauge-invariant threedimensional theory, with gauge fixed action

$\mathcal{S}_{\mathrm{RQED}_{3}}=\int \mathrm{d}^{3} x\left[\frac{1}{2} F^{\mu \nu} \frac{1}{\sqrt{-\partial^{2}}} F_{\mu \nu}+\bar{\psi}(i D) \psi+\frac{1}{2 \zeta}(\partial \cdot A)^{2}\right]$,

after the introduction of a new and now three-dimensional Abelian gauge field that, with a slight abuse of notation, we again call $A_{\mu}$. We have also added dynamics for the fermions, still without mass for the moment. The physical content of the theory will be gauge invariant and thus independent of the chosen gauge; thus, the gauge fixing terms before and after the reduction do not need to be the same. We have opted here for a simple linear gauge fixing rather than the involved reduced nonlocal gauge fixing term in $[16,17]$. The gauge parameter $\zeta$ here also carries a dimension, unlike $\xi$ in (7). The renormalization properties of $\mathrm{RQED} \equiv \mathrm{RQED}{ }_{3}$ were discussed in $[40,41]$. It should be noted that (12) already generates at tree level a branch cut in the complex momentum plane in the photon propagator, with a branch point at $p^{2}=0$. It is exactly the presence of the $1 / \sqrt{-\partial^{2}}$ in the kinetic gauge term that also allows us to keep the electromagnetic coupling constant $e$ dimensionless, even in a (reduced) three-dimensional space-time. Indeed, the new gauge field $A_{\mu}$ still has mass dimension 1, while for standard $\mathrm{QED}_{3}$ that mass dimension would amount to $1 / 2$. The nonlocal operator $\sqrt{-\partial^{2}}-1$ is to be understood via its three-dimensional Fourier (momentum) space representation [16]

$\frac{1}{\sqrt{-\partial^{2}}}\left(\vec{x}-\vec{x}^{\prime}\right)=\int \frac{d^{3} k}{2 \pi^{3}} \frac{e^{i \vec{k} \cdot\left(\vec{x}-\vec{x}^{\prime}\right)}}{k}, \quad k=\sqrt{\vec{k}^{2}}$.

If we add a Euclidean CS term, $i \theta \int \mathrm{d}^{3} x \epsilon_{\mu \nu \rho} A_{\mu} \partial_{\nu} A_{\rho}$, to the action in (12), we can deduce the tree-level photon propagator for a reduced Maxwell-CS theory, namely,

$$
\begin{aligned}
\hat{D}_{\mu \nu}(\vec{p})= & \frac{1}{2 p} \frac{1}{\left(1+\theta^{2}\right)}\left(\delta_{\mu \nu}-\frac{p_{\mu} p_{\nu}}{p^{2}}\right)-\frac{1}{2 p^{2}} \frac{\theta}{\left(1+\theta^{2}\right)} \epsilon_{\mu \nu \rho} p^{\rho} \\
& +\frac{\zeta}{p^{2}} \frac{p_{\mu} p_{\nu}}{p^{2}}
\end{aligned}
$$

From the CS term, we can infer that $\theta$ here is actually a dimensionless parameter, so unlike in standard $\mathrm{QED}_{3}$, it does not provide the theory with a "topological photon 
mass." This is consistent with the observation that RQED is scale invariant up to at least two loops; i.e., the beta function of the electromagnetic coupling vanishes [40,42]. On the other hand, $\theta \neq 0$ influences the photon propagator, not only by the presence of a T-odd contribution, but also by a normalization of the photon propagator. Intuitively, this corresponds to a down-scaling of the strength of the photon propagator, an effect not unlike increasing the mass of the exchanged particle.

\section{ONE-LOOP EXACTNESS OF TOPOLOGICAL PHOTON TERM IN REDUCED PLANAR QED: LORENTZ-INVARIANT CASE}

Our aim now is to prove that there will be no T-odd contributions to the gauge sector, i.e., the CS term, coming from radiative corrections beyond one loop. In principle, for the sake of physical interest, we could also try to add a generic electromagnetic background field to the action- to $\mathrm{QED}_{4}$ or $\mathrm{RQED}{ }_{3}$ - via the gauge principle of minimal coupling with the fermion fields. Background fields must be treated classically and, in the same way as the gauge sector, they must be defined in four dimensions. For possible interesting physics involving background fields, see for instance [43-45], including in-plane fields, which were also considered in [46]. For example, minimal coupling means we replace the covariant derivative in (12) as follows:

$$
i \not D \rightarrow i D+i \bar{A}_{0} \gamma^{0}+i \bar{A}_{3} \gamma^{3},
$$

where the barred gauge fields are classical in nature. Here $\bar{A}_{0}$ can describe a potential (electric field $\vec{E}$ ) applied in or orthogonal to the graphene sheet, while $\bar{A}_{3}$ can be used to couple an in-plane magnetic field $\vec{B} \| \vec{e}_{1}$. We remark here that the fields $A_{\mu}$ can be considered as the quantum fluctuations around these classical background fields $\bar{A}_{\mu}$. Taking the nonrelativistic limit of the corresponding Dirac equation, the latter coupling will provide the necessary magnetic field-magnetic moment coupling relevant for the Zeeman term considered in [46]. It is important to realize that although graphene is a sheet and the fermions will have no classical dynamics outside of the plane due to an inplane magnetic field, there is still the option for further quantum effects in the plane. Unfortunately, the tensorial basis elements relevant for the construction of a transverse self-energy, which play an important role in the ColemanHill argument, become far more complicated in the presence of background fields, mostly due to the increased number of allowed transverse tensors in Fourier (momentum) space. Moreover, nonconstant background fields make the situation utterly difficult. In light of this, we ignore background fields from our analysis in the current paper, and from now on we work with

$$
\begin{aligned}
\mathcal{S}_{\mathrm{RQED}_{3}}= & \int \mathrm{d}^{3} x\left[\frac{1}{2} F^{\mu \nu} \frac{1}{\sqrt{-\partial^{2}}} F_{\mu \nu}\right. \\
& \left.+\bar{\psi}\left(i D+m \gamma^{3}+m_{o} \gamma^{3} \gamma^{5}\right) \psi+\frac{1}{2 \zeta}(\partial \cdot A)^{2}\right] .
\end{aligned}
$$

Notice that $\vec{E} \cdot \vec{B}$ would be another T-odd scalar quantity, if present. In the absence of such fields we have allowed for the Haldane mass as another source of T-odd physics. As explained before, we opt for the $m \bar{\psi} \gamma^{3} \psi$-representation of the Dirac mass, although the following argument does not depend on which fermion masses are present; the actual numbers can, however.

\section{A. All order proof based on Ward identity}

First, we use the power of the global Ward identity associated with charge conservation to prove that (16) generates a CS term for the photon at one-loop order, or not at all. It is important that the fermions are massive to avoid spurious infrared singularities, so we can hereafter safely consider zero momentum expansions. Such an approach was suggested in [47] for standard $\mathrm{QED}_{3}$ while avoiding the combinatorial elements of the original proof of [25]. We follow as closely as possible the analysis of [47], paying attention to some differences where necessary.

We decompose, in Fourier space, the three-dimensional photon 1PI propagator (self-energy) in its most general form in a linear covariant gauge that is compatible with all Ward (Slavnov-Taylor) identities,

$$
\begin{aligned}
\hat{\Pi}_{\mu \nu}(\vec{p}) & =\left\langle\hat{A}_{\mu}(\vec{p}) \hat{A}_{\nu}(-\vec{p})\right\rangle^{1 P I} \\
& =\left(\delta_{\mu \nu}-\frac{p_{\mu} p_{\nu}}{p^{2}}\right) \Pi\left(p^{2}\right)+\epsilon_{\mu \nu \rho} p^{\rho} \vartheta\left(p^{2}\right) .
\end{aligned}
$$

Although it is well known that the photon self-energy is transverse, let us briefly review the underlying argument, as we will also need it later when we study the Lorentznoninvariant case (without change). It is most easily derived by replacing the action (16) by its equivalent version

$$
\begin{aligned}
\mathcal{S}_{\mathrm{RQED}_{3}}= & \int \mathrm{d}^{3} x\left[\frac{1}{2} F^{\mu \nu} \frac{1}{\sqrt{-\partial^{2}}} F_{\mu \nu}\right. \\
& +\bar{\psi}\left(i D+i A_{s} \gamma^{s}+m \gamma^{3}+m_{o} \gamma^{3} \gamma^{5}\right) \psi+b(\partial \cdot A) \\
& \left.-\frac{\zeta}{2} b^{2}+\bar{c} \partial^{2} c\right],
\end{aligned}
$$

including the multiplier $b$ and Faddeev-Popov (anti)ghost $\bar{c}, c$. Then the action (18) has a manifest BRST invariance, generated by 


$$
\begin{aligned}
s A_{\mu} & =-\partial_{\mu} c, \quad s \bar{c}=b, \quad s c=0, \quad s b=0, \\
s \psi & =-i e c \psi, \quad s \bar{\psi}=-i e \bar{\psi} c, \quad s^{2}=0 .
\end{aligned}
$$

We can define the composite operators $s \psi$ and $s \bar{\psi}$ at the quantum level by means of appropriate external sources coupling them to the theory,

$$
\Sigma=\mathcal{S}_{\mathrm{RQED}_{3}}+\int \mathrm{d}^{3} x[\overline{\mathcal{J}} s \psi-s \bar{\psi} \mathcal{J}] .
$$

At the functional level, the BRST invariance is encoded in

$$
\int \mathrm{d}^{3} x\left[-\partial_{\mu} c \frac{\delta \Sigma}{\delta A_{\mu}}+b \frac{\delta \Sigma}{\delta \bar{c}}+\frac{\delta \Sigma}{\delta \overline{\mathcal{J}}} \frac{\delta \Sigma}{\delta \psi}-\frac{\delta \Sigma}{\delta \mathcal{J}} \frac{\delta \Sigma}{\delta \bar{\psi}}\right]=0,
$$

which becomes the Slavnov-Taylor identity at the quantum level,

$$
\int \mathrm{d}^{3} x\left[-\partial_{\mu} c \frac{\delta \Gamma}{\delta A_{\mu}}+b \frac{\delta \Gamma}{\delta \bar{c}}+\frac{\delta \Gamma}{\delta \overline{\mathcal{J}}} \frac{\delta \Gamma}{\delta \psi}-\frac{\delta \Gamma}{\delta \mathcal{J}} \frac{\delta \Gamma}{\delta \bar{\psi}}\right]=0 .
$$

Here, $\Gamma$ is the quantum effective action, viz. the generating functional for the $1 P I$ correlation functions. We have also suppressed the space-time variable $x$ to avoid notational clutter. Acting with the test operator $\frac{\delta^{2}}{\delta c \delta A_{\nu}}$ on (22) and setting all external fields and sources to null, we indeed obtain the well-known transversality constraint

$$
\partial_{\mu} \frac{\delta^{2} \Gamma}{\delta A_{\mu} \delta A_{\nu}} \equiv \partial_{\mu} \Pi^{\mu \nu}=0
$$

Returning to the decomposition (17), the Coleman-Hill theorem now states that $\lim _{p^{2} \rightarrow 0} \vartheta\left(p^{2}\right)$ is solely determined by one-loop corrections.

To show this explicitly, we start from the path integral,

$$
\mathcal{I}=\int[\mathrm{d} \bar{\psi}][\mathrm{d} \psi]\left[\mathrm{d} A_{\mu}\right] e^{-\mathcal{S}_{\mathrm{RQED}_{3}},}
$$

with $\mathcal{S}_{\mathrm{RQED}_{3}}$ defined in (16). Then, diagrammatically, it is easily seen that at zero momentum, the graphs contributing to $\hat{\Pi}_{\mu \nu}\left(p^{2}\right)$ correspond to those of the 1 PI current-current correlator with zero momentum flow. We hence focus our attention on $\left\langle j_{\mu}(x) j_{\nu}(y)\right\rangle^{1 P I}$ and show that, at zero momentum, it is fully determined at one-loop order.

Classically, we can couple the current $j^{\mu}(x)$ to the action via an extra local source $\eta_{\mu}(x)$ by considering

$$
\Sigma^{\prime}=\Sigma+\int \mathrm{d}^{3} x \eta_{\mu} j^{\mu}
$$

Then

$$
\partial_{\mu} \frac{\delta \Sigma^{\prime}}{\delta \eta_{\mu}}=\bar{\psi} \frac{\delta \Sigma^{\prime}}{\delta \bar{\psi}}+\frac{\delta \Sigma^{\prime}}{\delta \psi} \psi
$$

expresses that the current is conserved. This is nothing other than the Noether theorem in functional language. As before, we get the quantum Ward identity

$$
\partial_{\mu} \frac{\delta \Gamma}{\delta \eta_{\mu}}=\bar{\psi} \frac{\delta \Gamma}{\delta \bar{\psi}}+\frac{\delta \Gamma}{\delta \psi} \psi .
$$

Here, we tacitly ignore the presence of the nonlocal operator $\frac{1}{\sqrt{-\partial^{2}}}$ as, strictly speaking, the quantum validity of Ward identities is only ensured in terms of local quantum field theory [48]. That being said, the nonlocality can be reduced from $\frac{1}{\sqrt{-\partial^{2}}}$ to $\sqrt{-\partial^{2}}$ by introducing an auxiliary antisymmetric tensor field $B_{\mu \nu}$ that allows us to replace $\int \mathrm{d}^{3} x \frac{1}{2} F^{\mu \nu} \frac{1}{\sqrt{-\partial^{2}}} F_{\mu \nu} \rightarrow \int \mathrm{d}^{3} x\left[\frac{1}{2} B^{\mu \nu} \sqrt{-\partial^{2}} B_{\mu \nu}+B_{\mu \nu} F^{\mu \nu}\right]$. This replacement does not affect the rest of the proof in this section, but a complete localization remains impossible unless we go back to a higher dimension of course, i.e., the original starting point with its mixed-dimensional action. However, it is also important to realize that the nonlocal term only affects the photon propagator, making it even softer in the infrared than for standard $\mathrm{QED}_{3}$. As such, the infrared is safer than expected, while for the interaction terms (leading to the nontrivial Feynman diagrams), standard power counting will apply. This is also the reason we could present the current analysis, which makes it clear that the RQED situation is, although similar, not completely the same as its better known cousin $\mathrm{QED}_{3}$, as treated in [47]. Power counting renormalizability of RQED was discussed in $[40,42,49]$. This ensures that the tree-level nonlocality will not spread into the theory; in that sense, there is no need to introduce more and more interaction vertices into the theory to maintain renormalizability. For example, if higher powers of $\frac{1}{\sqrt{-\partial^{2}}}$ are to be combined with higher powers of $F_{\mu \nu}$, we still have $d=3$, but higher order interaction vertices could appear radiatively. This is not the case for RQED. As $\operatorname{dim}\left[\eta_{\mu}\right]=1$, the quantum version of the action (25), which should contain all possible integrated $d=3$ polynomials of fields and sources compatible with the Ward identity constraints, will not be deformed by terms containing $\eta_{\mu}^{2}$ or higher powers, as no such terms can be constructed. The latter terms, if present, are responsible for contact terms in correlation functions containing the (gauge-invariant) current (see e.g., [50] for a similar observation). Thus, we do not need to worry about contact terms from the start, thereby evading the comment of [47].

Let us now denote with $\mathcal{V}_{0} \equiv-i \int \mathrm{d}^{3} x \bar{\psi} A_{\mu} \gamma^{\mu} \psi$ the standard gauge-boson fermion vertex operator. Then we can infer from the Ward identity (27) that

$$
\partial^{\mu}\left\langle j_{\mu}(x) j_{\nu}(y) \mathcal{V}_{0}^{n}\right\rangle^{1 P I}=0
$$


by taking another functional derivative of (27) w.r.t. $\eta_{\nu}(y)$, followed by $n \geq 0$ derivatives w.r.t. the coupling constant $e$ and setting all external sources and fields to zero at the end. The coupling $e$ acts as the (constant) source defining, by its derivatives, the quantum insertion of the vertex operator $\mathcal{V}_{0}$. Notice that each power of $\mathcal{V}_{0}$ is an integrated operator insertion, that is, one with zero momentum flow. Since (28) holds for any $n$ and since any expectation value of operators evaluated with the path integral partition function (24) can be succinctly rewritten as

$$
\left\langle j_{\mu}(x) j_{\nu}(y)\right\rangle_{\mathcal{S}_{\mathrm{RQED}_{3}}^{1 P I}}^{1 P}=\sum_{n \in \mathbb{N}}\left\langle j_{\mu}(x) j_{\nu}(y) e^{n} \mathcal{V}_{0}^{n}\right\rangle_{\text {quad }}^{1 P I},
$$

where "quad" refers to the quadratic (free theory) approximation of $\mathcal{S}_{\mathrm{RQED}_{3}}$, we can equally well write

$$
\sum_{n \in \mathbb{N}} \partial^{\mu}\left\langle j_{\mu}(x) j_{\nu}(y) e^{n} \mathcal{V}_{0}^{n}\right\rangle_{\text {quad }}^{1 P I}=0
$$

instead of (28).

For $n \geq 0$, each term in the expansion (29) can be expanded around zero momentum as

$$
\left\langle\hat{j}_{\mu}(p) \hat{j}_{\nu}(-p) \hat{\mathcal{V}}_{0}^{n}\right\rangle_{\text {quad }}^{1 P I}=a_{n} \delta_{\mu \nu}+b_{n} \epsilon_{\mu \nu \rho} p^{\rho}+\cdots
$$

after Fourier transforming. There is no need for the transverse projector $P_{\mu \nu}(p)=\delta_{\mu \nu}-\frac{p_{\mu} p_{\nu}}{p^{2}}$ here, as for $p \rightarrow 0$, this operator becomes proportional to $\delta_{\mu \nu}$.

Combining the constraint (30) with (31) then immediately gives $a_{n}=0$ for all $n \geq 0$. To control $b_{n}$, we use a small trick. We replace

$$
\begin{aligned}
& \left\langle\hat{j}_{\mu}(p) \hat{j}_{\nu}(-p) \hat{\mathcal{V}}_{0}^{n}\right\rangle_{\text {quad }}^{1 P I} \\
& \quad \rightarrow \lim _{k \rightarrow 0}\left\langle\hat{j}_{\mu}(p+k / 2) \hat{j}_{\nu}(-p+k / 2) \hat{\mathcal{V}}_{0}^{n-1} \hat{\mathcal{V}}_{k}\right\rangle_{\text {quad }}^{1 P I}
\end{aligned}
$$

i.e., we let a small net momentum $k$ flow through one of the vertices, keeping total momentum conservation in mind of course. Strictly speaking, from the viewpoint of renormalization, we should introduce here another local source to define the nonintegrated quantum operator $-i \bar{\psi} A_{\mu} \gamma^{\mu} \psi$, thereby deforming the original action (25) again. However, since we are only interested in the zero momentum limit, i.e., integrated operator, we refrain from doing so. This means we must exclude the $n=0$ term as we need at least one vertex insertion. Due to the symmetry $(\mu, p) \leftrightarrow$ $(\nu,-p)$ present in expression (32), only the following expansion can hold at leading order in $(p, k)$,

$$
\begin{aligned}
& \left\langle\hat{j}_{\mu}(p+k / 2) \hat{j}_{\nu}(-p+k / 2) \hat{\mathcal{V}}_{0}^{n-1} \hat{\mathcal{V}}_{k}\right\rangle_{\text {quad }}^{1 P I} \\
& \quad=A_{n} \delta_{\mu \nu}+B_{n} \epsilon_{\mu \nu \rho} p_{\rho}+\cdots
\end{aligned}
$$

Since $k$ does not appear in the foregoing expression, we actually have $B_{n}=b_{n}$ for $n \geq 1$ from the identification (32) together with the expansion (31). This aforementioned symmetry is crucial to avoid other possible momentum combinations in (33); we assume that [47] used the same observation, without having made it explicit.

The Fourier version of the constraint ${ }^{1}$ (30) now reads

$$
(p+k / 2)^{\mu}\left\langle\hat{j}_{\mu}(p+k / 2) \hat{j}_{\nu}(-p+k / 2) \hat{\mathcal{V}}_{0}^{n-1} \hat{\mathcal{V}}_{k}\right\rangle_{\text {quad }}^{1 P I}=0 .
$$

Applying this to (33) leads, next to $A_{n}=0$, to $b_{n}=0$ for all $n \geq 1$.

Putting everything back together, we have actually shown that

$$
\left\langle\hat{j}_{\mu}(p) \hat{j}_{\nu}(-p)\right\rangle_{\mathcal{S}_{\mathrm{RQED}_{3}}^{1 P I}}^{P I}=b_{0} \epsilon_{\mu \nu \rho} p_{\rho}+\mathcal{O}\left(p^{2}\right),
$$

which is nothing other than the Coleman-Hill theorem for RQED, as the corresponding zeroth order diagram contributing to (35) is equivalent to the one-loop photon selfenergy correction.

\section{B. Four-component vs two-component spinors}

As we mentioned in Sec. II, there are several theoretical instances to create a mass gap in the Dirac regime of graphene $\pi$-electrons, even if experimentally it is still very difficult to open a mass gap in a controllable way [15] (see $[14,33,51]$ for a detailed description of the different mass terms and their corresponding symmetry breaking). Here, we briefly survey how those mass terms are reduced in the four- and two-component spinor descriptions for these electrons. If we consider four-spinors in $(2+1)$ dimensions, the Lorentz generators are in a reducible $4 \times 4$ matrix representation $[14,27,31]$. We arrange the sublattice annihilation operators ( $a$ and $b$ ) with their corresponding valley numbers (subscripts + and - ) as

$$
\psi_{+}=\left(\begin{array}{c}
a_{+} \\
b_{+}
\end{array}\right), \quad \psi_{-}=\left(\begin{array}{c}
b_{-} \\
a_{-}
\end{array}\right),
$$

in the two-component representation, and as

$$
\psi=\left(\begin{array}{c}
\psi_{+} \\
\psi_{-}
\end{array}\right)
$$

in the case of a four-component representation.

As in the four-component description, we have at our disposal two matrices which anticommute with respect to

\footnotetext{
${ }^{1}$ This condition also holds when the operators $\hat{\mathcal{V}}_{k}$ are not integrated; this can be shown by coupling the operator $\bar{\psi} A \psi$ to the action $\Sigma$ with another local source and by manipulating the corresponding Ward identity.
} 
the rest $\left(\gamma^{3}\right.$ and $\left.\gamma^{5}\right)$; we basically have four kinds of masses. Notice that we do not consider the internal spin- $1 / 2$ nature of the $\pi$ electrons. By considering it, the number of mass terms increases considerably [33]. A standard mass term in four-component spinor language is of the form $m \bar{\psi} \psi=m \psi^{\dagger} \gamma^{0} \psi$, which breaks both symmetries (6a) and (6b), but it does not break time reversal symmetry in the four-dimensional matrix representation. This term mixes the flavors + and - ,

$$
S_{\text {usual }}=-\int \mathrm{d}^{3} x m \bar{\mu} \psi=-\int \mathrm{d}^{3} x m\left(\psi_{+}^{\dagger} \psi_{-}+\psi_{-}^{\dagger} \psi_{+}\right) .
$$

The mass terms considered in Sec. II, i.e., $i m \bar{\psi} \gamma^{5} \psi$ and $m \bar{\psi} \gamma^{3} \psi$, break one of the extended symmetries, (6a) and (6b), respectively, but preserve time reversal symmetry in the four-dimensional matrix representation. The first case is related to the Kekule distortion [52], while we can see that the second one allows us to rewrite the action in a twocomponent spinor decomposition as

$$
S_{\gamma^{3}}=-\int \mathrm{d}^{3} x m \bar{\psi} \gamma^{3} \psi=-\int \mathrm{d}^{3} x m\left(\psi_{+}^{\dagger} \sigma^{3} \psi_{+}-\psi_{-}^{\dagger} \sigma^{3} \psi_{-}\right) .
$$

We call this term the "normal" mass, as it is the usual mass for a two-component spinor in $(2+1)$ dimensions with two different decoupled flavors + and - .

The last possibility is the Haldane mass term [36], which does not break the symmetries (6a) and (6b) but does break time reversal symmetry [14]. This term also admits a decoupled two-component spinor decomposition,

$$
\begin{aligned}
S_{\text {Haldane }} & =-\int \mathrm{d}^{3} x m_{0} \bar{\psi} \gamma^{3} \gamma^{5} \psi \\
& =-\int \mathrm{d}^{3} x m_{o}\left(\psi_{+}^{\dagger} \sigma^{3} \psi_{+}+\psi_{-}^{\dagger} \sigma^{3} \psi_{-}\right) .
\end{aligned}
$$

We can see that the mass terms (38) and (39) have different relative sign for the two flavors + and - .

The CS mass term can be generated by T-odd fermion one-loop corrections. These corrections at zero momentum are of the form $[35,38,53,54]$

$$
\Gamma_{\mu \nu}^{\text {odd }} \sim \frac{m}{|m|} \epsilon_{\mu \nu \rho} p_{\rho},
$$

implying that the term (38) will give a net zero contribution for the CS photon mass, while (39) does contribute. More precisely, we get, at the level of the action, a (exact) radiatively introduced $\mathrm{T}$-photon term

$$
S_{\mathrm{CS}}=\int \mathrm{d}^{3} x\left(-i \frac{e^{2}}{4 \pi} \frac{m_{o}}{\left|m_{o}\right|} \epsilon^{\mu \nu \rho} A_{\mu} \partial_{\nu} A_{\rho}\right)
$$

when a Haldane term (39) is coupled to RQED. Here, again, we note the role of the dimensionless coupling in RQED. Indeed, in the case of $\mathrm{QED}_{3}$ the factor $e^{2}$ in (41) is what "feeds" the dynamical topological photon mass $\theta$ thanks to $e^{2}$ having mass dimension 1 , whereas now the dimensionless nature of $e^{2}$ gives a dimensionless parameter $\theta$ in front of the CS term.

\section{ONE-LOOP EXACTNESS OF TOPOLOGICAL PHOTON TERM IN REDUCED PLANAR QED: LORENTZ-NONINVARIANT CASE}

Having proven the Coleman-Hill theorem in the case of Lorentz-invariant $\mathrm{RQED}_{3}$, let us now turn to the generalization in terms of the action [based on the one of (4), supplemented with the photon field and BRST-invariant linear gauge fixing]

$$
\begin{aligned}
\mathcal{S}_{\mathrm{RQED}_{3}}= & \int \mathrm{d}^{3} x\left[\frac{1}{2} F^{\mu \nu} \frac{1}{\sqrt{-\partial^{2}}} F_{\mu \nu}+\bar{\psi}\left(i \gamma^{0}\left(\partial_{0}+e A_{0}\right)\right.\right. \\
& \left.+i v_{F} \gamma^{i}\left(\partial_{i}+e A_{i}\right)+m \gamma^{3}+m_{o} \gamma^{3} \gamma^{5}\right) \psi \\
& \left.+b(\partial \cdot A)-\frac{\zeta}{2} b^{2}+\bar{c} \partial^{2} c\right]
\end{aligned}
$$

i.e., we take into account the Fermi velocity $v_{F}$. To avoid further notational clutter, we keep the notation $\mathcal{S}_{\mathrm{RQED}_{3}}$ for the classical action, $\Sigma$ for the classical action supplemented with external sources, and $\Gamma$ for the quantum effective action. We briefly note the points that do not change but go into more detail about the necessary, significant adaptations. Some quantum aspects of a similar theory-for massless fermions and within the approximation of an instantaneous Coulomb interaction-were discussed in [55], including the renormalization of $v_{F}$ away from the fixed point $v_{F}=1$ (also studied in [56]), the latter corresponding to the Lorentz-invariant limit. Dyson-Schwinger equation based studies are presented in e.g., [57,58].

Clearly, the action (42) is still BRST invariant w.r.t. (19), so the transversality constraint (23) holds, irrespective of $v_{F}$. As the Lorentz invariance is reduced to twodimensional rotational invariance in the $(1,2)$-plane, the tensorial decompositions used in Sec. III become a bit more elaborate. The self-energy can now be decomposed into

$$
\begin{aligned}
\Pi_{\mu \nu}(\vec{p})= & \left\langle A_{\mu}(\vec{p}) A_{\nu}(-\vec{p})\right\rangle^{1 P I} \\
= & P_{\mu \nu}^{1} \Pi_{1}\left(p_{0}, p_{i} p_{i}\right)+P_{\mu \nu}^{2} \Pi_{2}\left(p_{0}, p_{i} p_{i}\right) \\
& +\epsilon_{\mu \nu \rho} p_{\rho} \vartheta\left(p_{0}, p_{i} p_{i}\right) .
\end{aligned}
$$

We have introduced two transverse projectors, similar to what is known from finite-temperature field theory $[59,60]$, as the raison d'être for the relevance of these two tensors is the same: the 0 -direction is singled out as "special." To be more precise, we have, with $i, j \in\{1,2\}$, 


$$
\begin{aligned}
& P_{\mu \nu}^{(1)}= \begin{cases}0 & \mu=0 \text { or } \nu=0 \\
\delta_{i j}-\frac{p_{i} p_{j}}{p^{2}} & \text { otherwise, }\end{cases} \\
& P_{\mu \nu}^{(2)}=\left(\delta_{\mu \nu}-\frac{p_{\mu} p_{\nu}}{p^{2}}\right)-P_{\mu \nu}^{(1)}
\end{aligned}
$$

The decomposition (43) is the most general one that is compatible with the symmetry $(\mu, p) \leftrightarrow(\nu,-p)$, the twodimensional rotational invariance, and the transversality constraint $p^{\mu} \Pi_{\mu \nu}=0$. The form factors can depend separately on $p_{0}$ and $p_{i} p_{i}$, as indicated.

The (conserved) fermion current is now given by

$$
j_{\mu}=\bar{\psi} i \gamma^{0} \psi \delta_{\mu 0}+v_{F} \bar{\psi} i \gamma^{i} \psi \delta_{\mu i}, \quad \partial^{\mu} j_{\mu}=0,
$$

while the photon-fermion vertex becomes $\mathcal{V}_{0}=$ $-i \int \mathrm{d}^{3} x \bar{\psi} A_{0} \gamma^{0}-i v_{F} \int \mathrm{d}^{3} x \bar{\psi} A_{i} \gamma^{i}$. This vertex could be split into two vertices, but considering all powers of $\mathcal{V}_{0}$ in the series expansion will generate all necessary powers of its two substructures; thus, we can maintain a single vertex expression, for simplicity.

The connection between the self-energy $\Pi_{\mu \nu}(\vec{p})$ and the current-current correlator remains valid, so it is still sufficient to control the low momentum expansion of $\left\langle j_{\mu} j_{\nu}\right\rangle^{1 P I}$, which is rewritable as, using the same arguments as in Eq. (29),

$$
\left\langle j_{\mu} j_{\nu}\right\rangle_{\mathcal{S}_{\mathrm{RQED}_{3}}}^{P^{P I}}=\sum_{n \in \mathbb{N}}\left\langle j_{\mu} j_{\nu} e^{n} \mathcal{V}_{0}^{n}\right\rangle_{\text {quad }}^{1 P I}
$$

and subject to (30), with the appropriate vertex replacement.

A word of caution is needed here. As the fermions are massive, just as before, there will be no singular behavior around zero momentum, despite the lack of Lorentz invariance. This situation is in sharp contrast with finite temperature, where nonanalytic behavior emerges at zero momentum (see for instance $[60,61]$ ). In particular, the limits $p_{0} \rightarrow 0$ and $p_{i} \rightarrow 0$ do not commute, as they correspond to different physics. Intuitively, a finite-temperature medium opens extra reaction channels (particle absorption from the medium), leading to extra branch points, in particular, at the momentum origin [61]. This lies at the heart of the nonvalidity of the Coleman-Hill theorem at finite temperature, explicitly illustrated in [60]. Our current setup is inherently different, as we have no thermal medium. As such, for each $n \geq 0$, we can expand the terms in (46) as follows:

$$
\begin{aligned}
\left\langle\hat{j}_{\mu}(p) \hat{j}_{\nu}(-p) \hat{\mathcal{V}}_{0}^{n}\right\rangle_{\text {quad }}^{1 P I}= & a_{n} \delta_{\mu 0} \delta_{\nu 0}+a_{n}^{\prime} \delta_{\mu i} \delta_{\nu i} \\
& +b_{n} \epsilon_{\mu \nu \rho} p_{\rho}+\cdots
\end{aligned}
$$

The two delta terms correspond to the zero momentum limits of the aforementioned transverse projectors. The rest of the argument proceeds analogously as in Sec. III, eventually leading to $a_{n}=a_{n}^{\prime}=0, \forall n \geq 0$, and $b_{n}=0, \forall n \geq 1$. In other words, the zero momentum limit of the topological term in the photon self-energy is exact at one-loop order; i.e., the Coleman-Hill theorem applies to the theory described by the action (42).

It is useful to compute $\vartheta(0)$ and see how it depends on $v_{F}$. We follow $[35,62]$ and consider first a single twocomponent (Euclidean) spinor with standard Dirac mass. We use

$\gamma^{0}=\left(\begin{array}{cc}-i & 0 \\ 0 & i\end{array}\right), \quad \gamma^{1}=\left(\begin{array}{cc}0 & i \\ i & 0\end{array}\right), \quad \gamma^{2}=\left(\begin{array}{cc}0 & 1 \\ -1 & 0\end{array}\right)$.

The one-loop photon self-energy is then given by

$$
\begin{aligned}
\Pi_{\mu \nu}(\vec{p})= & e^{2} \int \frac{\mathrm{d}^{3} k}{(2 \pi)^{3}} \operatorname{Tr}\left[\left(\gamma^{0} \delta_{\mu 0}+v_{F} \gamma^{i} \delta_{\mu 0}\right)\right. \\
& \left.\times S_{F}(\vec{p}+\vec{k})\left(\gamma^{0} \delta_{\nu 0}+v_{F} \gamma^{j} \delta_{\nu j}\right) S_{F}(\vec{k})\right],
\end{aligned}
$$

with a fermion propagator reading

$$
S_{F}(\vec{p})=\frac{p_{0} \gamma^{0}+v_{F} p_{i} \gamma^{i}-m}{p_{0}^{2}+v_{F}^{2} p_{i} p_{i}} .
$$

To facilitate the computation, we can introduce $\vec{P}=$ $\left(p_{0}, v_{F} p_{i}\right)$, and doing the same for the integration momentum $\vec{K}=\left(k_{0}, v_{F} k_{i}\right)$, we get

$$
\begin{aligned}
\hat{\Pi}_{\mu \nu}(\vec{p}) \equiv & \tilde{\Pi}_{\mu \nu}(\vec{P}) \\
= & \frac{e^{2}}{v_{F}^{2}} \int \frac{\mathrm{d}^{3} K}{(2 \pi)^{3}} \operatorname{Tr}\left[\left(\gamma^{0} \delta_{\mu 0}+v_{F} \gamma^{i} \delta_{\mu 0}\right)\right. \\
& \left.\times \frac{P+K-m}{(P+K)^{2}+m^{2}}\left(\gamma^{0} \delta_{\nu 0}+v_{F} \gamma^{j} \delta_{\nu j}\right) \frac{K-m}{K^{2}+m^{2}}\right] .
\end{aligned}
$$

As we are only interested in the piece $\propto \epsilon_{\mu \nu \rho} p^{\rho}$, it is clear that the only relevant contributions to this odd piece come from combining an odd number of $\gamma$ matrices, based on the property $\operatorname{Tr}\left(\gamma^{\mu} \gamma^{\nu} \gamma^{\rho}\right)=-2 \epsilon^{\mu \nu \rho}$. It is clear from the above expression (51) that we will find for the integral, at leading order in $P$ and thus in $p$, the same result as if $v_{F}=1$, modulo the fact that each time a spatial index appears, an extra factor of $v_{F}$ is to be included, either coming from the $\gamma^{i}$-and/or $\gamma^{j}$-vertex, or from the spatial part of the $P_{\rho}$-factor multiplying $\epsilon_{\mu \nu \rho}$. Thanks to the $\epsilon$-symbol, we know that exactly two such spatial indices will appear in any case, so taking into account the prefactors of the integral, we ultimately find

$$
\hat{\Pi}_{\mu \nu}(\vec{p})=\frac{e^{2}}{4 \pi} \frac{m}{|m|} \epsilon_{\mu \nu \rho} p^{\rho}+\mathcal{O}\left(p^{2}\right)
$$

i.e., the topological photon term does not depend on the Fermi velocity. This result confirms the earlier finding of [63], where a $v_{F}$ rescaling of the spatial $\gamma$ matrices was 
introduced to facilitate the one-loop computation of the self-energy.

Returning to the graphene case with four-component spinors, we thus find no dynamically generated CS term in the presence of a Dirac mass, while a Haldane mass leads to

$$
S_{\mathrm{CS}}=\int \mathrm{d}^{3} x\left(-i \frac{e^{2}}{4 \pi} \frac{m_{o}}{\left|m_{o}\right|} \epsilon^{\mu \nu \rho} A_{\mu} \partial_{\nu} A_{\rho}\right)
$$

if the underlying dynamics is governed by the action (42).

\section{OUTLOOK}

We have shown that, in the framework of reduced QED in $(2+1)$ dimensions, the topological piece of the photon selfenergy at zero momentum only receives quantum corrections up to one loop. Using fundamental arguments based on the $U(1)$ Ward identity, we have proven that all two- and higherloop contributions are identically zero. In other words, besides holding for ordinary $\mathrm{QED}_{3}$, the Coleman-Hill theorem thus also applies in the case of a theory containing nonlocal terms in the action, where the gauge fields are not constrained to the plane but the fermions are; this is irrespective of the presence of the Fermi velocity $v_{F}<c$ which explicitly breaks the Lorentz invariance. Let us point out that Lorentz invariance can be broken in an even more severe way, namely, rotational symmetry breaking, once we abandon the linear regime due to the honeycomb lattice structure. This has important consequences for the $\pi$ electron description in such regimes [64,65]. For completeness, we have also derived the tree-level photon propagator for this theory, taking into account the CS term. Interestingly, for the RQED case, the parameter $\theta$ in front of the CS term is not a mass, as for $\mathrm{QED}_{3}$, but somehow acts as a dimensionless suppressing factor in the photon propagator [see (14)]. Moreover, we computed the exact value of $\theta$ in the case where the four-component Dirac fermions are massive for two different realizations of the mass term, both relevant for graphene studies [see (38) and (39)], which is valid for both Lorentz-invariant and noninvariant cases.
Our observations pave the road to more deeply investigate the interconnection between the CS photon term and Haldane fermion mass in the specific case of RQED. Any interaction term or fermion mass has a direct influence on the vector and axial current channels which, in the context of graphene physics, provide us with relevant observables for transport phenomena. A mapping between the two sectors of the theory would also allow us to investigate how the presence of external electromagnetic fields effectively manifest themselves in the fermion sector. An important piece of information will be encoded in the $\theta$-sector of the photon propagator, which we expect to be quite sensitive to such background fields. Numerical estimates for the influence of the CS term on the Haldane mass and/or $\gamma^{3}$-Dirac mass, making use of Dyson-Schwinger equations, along the same lines as the $\mathrm{QED}_{3}$ study of [66], are currently being prepared and will be reported in a forthcoming work, paying particular attention on the role of the Fermi velocity $v_{F}$. Moreover, we hope to establish the oneloop exactness of the topological photon term, at least in the case of constant background electric and magnetic fields, which are of phenomenological relevance, as outlined in our text. These backgrounds can be rephrased in terms of a single space-time dependent background gauge field, which itself remains constant in momentum space, up to appropriate (derivatives of) $\delta$ functions. It should hence be possible to construct the most general transverse tensor basis, in the presence of both aforementioned background fields and Fermi velocity $v_{F}$, and apply similar techniques as outlined here.

\section{ACKNOWLEDGMENTS}

We thank S. Hernández-Ortiz, A. Raya, and C. Villavicencio for useful correspondence. A. J. M. is a beneficiary of a grant from the Belgian Federal Science Policy (BELSPO) and receives partial support from FAPESP under Grant No. 2016/12705-7. The work of P. P. is supported by a PDM grant from KU Leuven.
[1] T. W. Appelquist, M. J. Bowick, D. Karabali, and L. C. R. Wijewardhana, Phys. Rev. D 33, 3774 (1986).

[2] T. W. Appelquist, M. J. Bowick, D. Karabali, and L. C. R. Wijewardhana, Phys. Rev. D 33, 3704 (1986).

[3] C. S. Fischer, R. Alkofer, T. Dahm, and P. Maris, Phys. Rev. D 70, 073007 (2004).

[4] A. Bashir, A. Raya, I. C. Cloet, and C. D. Roberts, Phys. Rev. C 78, 055201 (2008).

[5] V. P. Gusynin and P. K. Pyatkovskiy, Phys. Rev. D 94, 125009 (2016).
[6] T. O. Wehling, A. M. Black-Schaffer, and A. V. Balatsky, Adv. Phys. 63, 1 (2014).

[7] M. I. Katsnelson, K. S. Novoselov, and A. K. Geim, Nat. Phys. 2, 620 (2006).

[8] M. A. H. Vozmediano, M. I. Katsnelson, and F. Guinea, Phys. Rep. 496, 109 (2010).

[9] A. Cortijo, F. Guinea, and M. A. H. Vozmediano, J. Phys. A 45, 383001 (2012).

[10] M. N. Chernodub, A. Cortijo, A. G. Grushin, K. Landsteiner, and M. A. H. Vozmediano, Phys. Rev. B 89, 081407 (2014). 
[11] Q. Li, D. E. Kharzeev, C. Zhang, Y. Huang, I. Pletikosic, A. V. Fedorov, R. D. Zhong, J. A. Schneeloch, G. D. Gu, and T. Valla, Nat. Phys. 12, 550 (2016).

[12] A. Iorio and G. Lambiase, Phys. Lett. B 716, 334 (2012).

[13] A. Iorio and G. Lambiase, Phys. Rev. D 90, 025006 (2014).

[14] V. P. Gusynin, S. G. Sharapov, and J. P. Carbotte, Int. J. Mod. Phys. B 21, 4611 (2007).

[15] A. H. Castro Neto, F. Guinea, N. M. R. Peres, K. S. Novoselov, and A. K. Geim, Rev. Mod. Phys. 81, 109 (2009).

[16] E. C. Marino, Nucl. Phys. B408, 551 (1993).

[17] E. V. Gorbar, V. P. Gusynin, and V. A. Miransky, Phys. Rev. D 64, 105028 (2001).

[18] V. S. Alves, W. S. Elias, L. O. Nascimento, V. Juričić, and F. Peña, Phys. Rev. D 87, 125002 (2013).

[19] E. C. Marino, L. O. Nascimento, V.S. Alves, and C. M. Smith, Phys. Rev. D 90, 105003 (2014).

[20] E. C. Marino, L. O. Nascimento, V. S. Alves, and C. M. Smith, Phys. Rev. X 5, 011040 (2015).

[21] L. O. Nascimento, V. S. Alves, F. Peña, C. M. Smith, and E. C. Marino, Phys. Rev. D 92, 025018 (2015).

[22] S. Deser, R. Jackiw, and S. Templeton, Ann. Phys. (N.Y.) 140, 372 (1982); 281, 409 (2000).

[23] S. Deser, R. Jackiw, and S. Templeton, Phys. Rev. Lett. 48, 975 (1982).

[24] Y.-H. Chen, F. Wilczek, E. Witten, and B. I. Halperin, Int. J. Mod. Phys. B 03, 1001 (1989).

[25] S. R. Coleman and B. R. Hill, Phys. Lett. 159B, 184 (1985).

[26] V. P. Spiridonov and F. V. Tkachov, Phys. Lett. B 260, 109 (1991).

[27] S. Hands, J. High Energy Phys. 09 (2015) 047.

[28] R. Jackiw and S. Y. Pi, Phys. Rev. Lett. 98, 266402 (2007).

[29] K. S. Novoselov, A. K. Geim, S. V. Morozov, D. Jiang, M. I. Katsnelson, I. V. Grigorieva, S. V. Dubonos, and A. A. Firsov, Nature (London) 438, 197 (2005).

[30] C. Chamon, C.-Y. Hou, R. Jackiw, C. Mudry, S.-Y. Pi, and A. P. Schnyder, Phys. Rev. Lett. 100, 110405 (2008).

[31] A. Iorio, Int. J. Mod. Phys. D 24, 1530013 (2015).

[32] G. W. Semenoff, Phys. Rev. Lett. 53, 2449 (1984).

[33] S. Ryu, C. Mudry, C.-Y. Hou, and C. Chamon, Phys. Rev. B 80, 205319 (2009).

[34] R. Jackiw and S. Templeton, Phys. Rev. D 23, 2291 (1981).

[35] G. V. Dunne, arXiv:hep-th/9902115.

[36] F. D. M. Haldane, Phys. Rev. Lett. 61, 2015 (1988).

[37] R. Delbourgo and A. B. Waites, Phys. Lett. B 300, 241 (1993).

[38] R. Delbourgo and A. B. Waites, Aust. J. Phys. 47, 465 (1994).

[39] A. Bashir, A. Raya, and S. Sanchez-Madrigal, J. Phys. A 41, 505401 (2008).
[40] S. Teber, Phys. Rev. D 86, 025005 (2012).

[41] A. V. Kotikov and S. Teber, Phys. Rev. D 89, 065038 (2014).

[42] S. Teber and A. V. Kotikov, Phys. Rev. D 97, 074004 (2018).

[43] A. J. Mizher, A. Raya, and C. Villavicencio, Int. J. Mod. Phys. B 30, 1550257 (2016).

[44] A. J. Mizher, A. Raya, and C. Villavicencio, Nucl. Part. Phys. Proc. 270-272, 181 (2016).

[45] A. J. Mizher, S. Hernandez-Ortiz, A. Raya, and C. Villavicencio, arXiv:1803.05794.

[46] I. L. Aleiner, D. E. Kharzeev, and A. M. Tsvelik, Phys. Rev. B 76, 195415 (2007).

[47] T. Shacham, J. High Energy Phys. 05 (2013) 147.

[48] O. Piguet and S. P. Sorella, Lect. Notes Phys., M: Monogr. 28, 1 (1995).

[49] C. P. Herzog and K.-W. Huang, J. High Energy Phys. 10 (2017) 189.

[50] D. Dudal, S. P. Sorella, N. Vandersickel, and H. Verschelde, Eur. Phys. J. C 64, 147 (2009).

[51] Y. Concha-Sanchez, A. Raya, and M. E. Tejeda-Yeomans, Phys. Rev. D 87, 035001 (2013).

[52] C.-Y. Hou, C. Chamon, and C. Mudry, Phys. Rev. Lett. 98, 186809 (2007).

[53] A. N. Redlich, Phys. Rev. Lett. 52, 18 (1984).

[54] A. N. Redlich, Phys. Rev. D 29, 2366 (1984).

[55] D. T. Son, Phys. Rev. B 75, 235423 (2007).

[56] I. F. Herbut, V. Juricic, and B. Roy, Phys. Rev. B 79, 085116 (2009).

[57] M. E. Carrington, C. S. Fischer, L. von Smekal, and M. H. Thoma, Phys. Rev. B 94, 125102 (2016).

[58] M. E. Carrington, C. S. Fischer, L. von Smekal, and M. H. Thoma, Phys. Rev. B 97, 115411 (2018).

[59] J. I. Kapusta and C. Gale, Finite-Temperature Field Theory: Principles and Applications, Cambridge Monographs on Mathematical Physics (Cambridge University Press, Cambridge, England, 2011).

[60] F. T. Brandt, A. K. Das, and J. Frenkel, Phys. Lett. B 492, 393 (2000).

[61] A. K. Das, Finite Temperature Field Theory (World Scientific, New York, 1997).

[62] M. Chaichian, W. F. Chen, and H. C. Lee, Phys. Lett. B 409, 325 (1997).

[63] I. V. Fialkovsky and D. V. Vassilevich, Mod. Phys. Lett. A 31, 1630047 (2016).

[64] A. Iorio and P. Pais, Phys. Rev. D 92, 125005 (2015).

[65] A. Iorio, P. Pais, I. A. Elmashad, A. F. Ali, M. Faizal, and L. I. Abou-Salem, Int. J. Mod. Phys. D 27, 1850080 (2018).

[66] C. P. Hofmann, A. Raya, and S. S. Madrigal, Phys. Rev. D 82, 096011 (2010). 